\section{Binding of 14-3-3 proteins and nuclear export control the intracellular localization of the mitotic inducer Cdc25}

\author{
Akiko Kumagai and William G. Dunphy ${ }^{1}$ \\ Division of Biology 216-76, Howard Hughes Medical Institute, \\ California Institute of Technology, \\ Pasadena, California 91125 USA
}

Binding of 14-3-3 proteins near the nuclear localization sequence of Xenopus Cdc25 suppresses its ability to induce entry into mitosis. We have examined the intracellular localization of green fluorescent protein (GFP)tagged wild-type Cdc25 or a mutant (S287A) that cannot bind 14-3-3 proteins. Upon coexpression with Myc-143-3€, GFP-Cdc25-WT was predominantly cytoplasmic, whereas GFP-Cdc25-S287A was exclusively nuclear. Leptomycin B, an inhibitor of nuclear export, elicited a prompt redistribution of GFP-Cdc25-WT to the nucleus. Mutagenesis experiments demonstrated that Cdc25 contains multiple nuclear export sequences. These studies indicate that the binding of 14-3-3 proteins and nuclear export regulate the intracellular localization of Cdc25.

Received January 12, 1999; revised version accepted March 23,1999

The entry into mitosis is triggered by maturation-promoting factor (MPF), which contains the protein kinase Cdc2, a B-type mitotic cyclin, and a Suc1/Cks protein (Morgan 1997). In higher eukaryotes, the activity of Cdc2 is suppressed during interphase by inhibitory phosphorylations on Thr-14 and Tyr-15 (Morgan 1997). The activation of MPF at the $\mathrm{G}_{2} / \mathrm{M}$ boundary occurs when the dual-specificity phosphatase Cdc25C dephosphorylates these sites (Dunphy and Kumagai 1991; Gautier et al. 1991).

The action of Cdc25C is strictly regulated during the cell cycle (Izumi et al. 1992; Kumagai and Dunphy 1992). Cdc25C is activated at mitosis by a kinase cascade containing Cdc2/cyclin B itself and Plx1 (Hoffmann et al. 1993; Izumi and Maller 1995; Kumagai and Dunphy 1996). Furthermore, during interphase, Cdc25C is suppressed by the binding of inhibitory 14-3-3 proteins (Peng et al. 1997; Kumagai et al. 1998b). 14-3-3 proteins bind to a critical phosphoserine (Ser-216 and Ser-287 in human and Xenopus Cdc25C, respectively) after phosphorylation by Chk1 and other kinases (Furnari et al. 1997; Peng et al. 1997; Sanchez et al. 1997; Kumagai et al. 1998a; Matsuoka et al. 1998). The association between 14-3-3

[Key Words: Cdc2; cell cycle; mitosis; Chk1; phosphorylation] ${ }^{1}$ Corresponding author.

E-MAIL dunphy@cco.caltech.edu; FAX (626) 795-7563. and Cdc25 is ultimately reversed at mitosis but is maintained in the presence of unreplicated or damaged DNA. The binding of 14-3-3 has little or no effect on the phosphatase activity of Cdc25C, suggesting that 14-3-3 proteins do not directly affect catalysis (Peng et al. 1997; Kumagai et al. 1998b; Blasina et al. 1999).

In Xenopus Cdc25C, the binding site for 14-3-3 around Ser-287 is immediately adjacent to a putative bipartite nuclear localization sequence (NLS) at residues 298-316, suggesting a mechanism whereby 14-3-3 proteins could influence the intracellular localization of this regulatory phosphatase. It has been shown that cyclin B1 shuttles dynamically between the cytoplasm and nucleus and that this trafficking is important for proper cell cycle control (Hagting et al. 1998; Jin et al. 1998; Toyoshima et al. 1998; Yang et al. 1998). Coordinated localization of Cdc25C and Cdc2/cyclin B may be required for faithful mitotic regulation.

\section{Results}

To assess whether the binding of 14-3-3 proteins has a role in the localization of Xenopus Cdc25C (Cdc25), we expressed hybrid proteins consisting of green fluorescent protein (GFP) and either wild-type Cdc25 (GFP-Cdc25WT) or a mutant of Cdc25 that cannot bind 14-3-3 (GFPCdc25-S287A) in transfected Xenopus tissue culture (XTC) cells. As shown in Figure 1, in cells expressing low to moderate levels of GFP-Cdc25-WT, the protein was mainly cytoplasmic. However, in cells expressing high levels of GFP-Cdc25-WT, the protein could be found in both the cytoplasmic and nuclear compartments. In contrast, the GFP-Cdc25-S287A mutant, which cannot associate with 14-3-3 (Kumagai et al. 1998b), was almost exclusively nuclear at both low and high levels of expression. In cells expressing GFP alone (GFP is a $27-\mathrm{kD}$ protein that should readily traverse the nuclear pore), the fluorescent signal was distributed throughout the nucleus and cytoplasm at all levels of expression (not shown). By immunoblotting XTC cell lysates with antiCdc25 antibodies, we established that both GFP-Cdc25WT and GFP-Cdc25-S287A had not undergone detectable proteolysis, indicating that the fluorescent signal from GFP accurately reflects the localization of the hybrid proteins (not shown).

To address the mechanism by which Cdc25 enters the nucleus, we mutated its putative bipartite NLS (298KRPVRPLDSETPVRVKRRR-316) by deleting residues 311-316 to create the Cdc25- - NLS mutant. As shown in Figure 1, the GFP-Cdc25-S287A- $\Delta$ NLS protein in transfected cells is exclusively cytoplasmic. The same results were obtained for the GFP-Cdc25- $\Delta$ NLS protein (not shown). Thus, the 298-316 segment is essential for Xenopus Cdc25 to accumulate in the nucleus.

The nuclear accumulation of GFP-Cdc25-S287A suggested that the binding of 14-3-3 might promote the cytoplasmic retention and/or nuclear exclusion of wildtype Cdc25. However, it was also clear that GFP-Cdc25- 


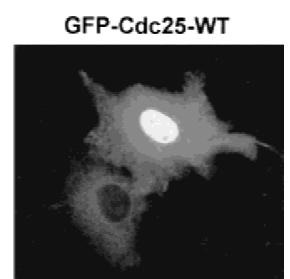

GFP-Cdc25-S287A

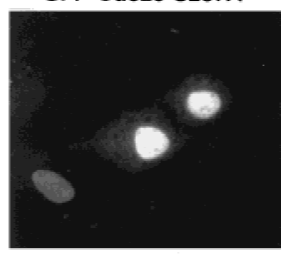

GFP-Cdc25-S287A- $\Delta$ NLS

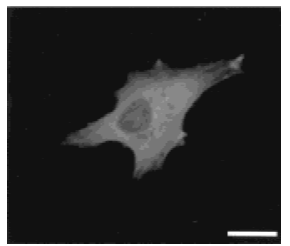

Figure 1. Localization of the GFP-Cdc25-WT, GFP-Cdc25S287A, and GFP-Cdc25-S287A- - NLS proteins in XTC cells. Cells were transfected with plasmids encoding GFP-Cdc25-WT (top), GFP-Cdc25-S287A (middle), or GFP-Cdc25-S287A$\triangle$ NLS (bottom) and observed by fluorescence microscopy. Magnification bar, $20 \mu \mathrm{m}$.

WT can be found in the nucleus, especially when this protein is expressed at high levels. We considered the possibility that, at high levels of GFP-Cdc25-WT, the supply of 14-3-3 protein in the XTC cells might be limiting. To address this issue, we cotransfected the cells with a construct that could express a Myc-tagged version of Xenopus 14-3-3€, which is the main partner of Cdc25 (Kumagai et al. 1998b). We observed that coexpression of Myc-14-3-3 $\epsilon$ markedly affected the intracellular distribution of GFP-Cdc25-WT (Fig. 2A,G). In particular, GFP-Cdc25-WT was principally cytoplasmic in the presence of exogenously expressed Myc-14-3-3€. In the presence of Myc-14-3-3€, a similar distribution of GFPCdc25-WT was observed when the XTC cells were irradiated with ultraviolet light at a dose of $40 \mathrm{~J} / \mathrm{m}^{2}$ (not shown). Cotransfection with a vector expressing the Myc tag alone was not able to promote localization of GFPCdc25-WT to the cytoplasm (Fig. 2G). Furthermore, Myc-14-3-3€ did not affect the predominantly nuclear localization of the GFP-Cdc25-S287A mutant that cannot associate with 14-3-3€ (Fig. 2B,G).

The cytoplasmic localization of GFP-Cdc25-WT in cells coexpressing Myc-14-3-3€ could mean that Cdc25 cannot enter the nucleus efficiently when bound to 143-3. A related, but not mutually exclusive, possibility is that binding of 14-3-3 might promote the elimination of Cdc25 from the nuclear compartment. To explore this issue, we used leptomycin B (LMB), an agent that blocks nuclear export. This drug inhibits Crm1/Exportin 1, a receptor that mediates the nuclear extrusion of proteins containing a nuclear export sequence (NES) (Fornerod et al. 1997; Fukuda et al. 1997; Ossareh-Nazari et al. 1997). Treatment of XTC cells with LMB led to a significant redistribution of GFP-Cdc25-WT within $2 \mathrm{hr}$ from a mainly cytoplasmic to an almost exclusively nuclear localization (Fig. 2C). Myc-14-3-3€ was mainly cytoplasmic in the absence of LMB (Fig. 2D). In the presence of LMB, a portion of the Myc-14-3-3€ accumulated in the nucleus, where at least some of the Myc-14-3-3€ is presumably bound to GFP-Cdc25-WT (Fig. 2F). In control experiments, we showed by immunoprecipitation of XTC cell lysates with anti-Cdc25 antibodies that GFPCdc25-WT and Myc-14-3-3€ remained associated with one another in the presence of LMB (not shown), indicating that LMB does not act indirectly by promoting reactions that lead to the dissociation of $14-3-3 \epsilon$ from Cdc25.

NESs typically contain a number of closely spaced leucines with the consensus Leu-(2-3X)-Leu- $(2-3 X)$-Leu- $X$ -

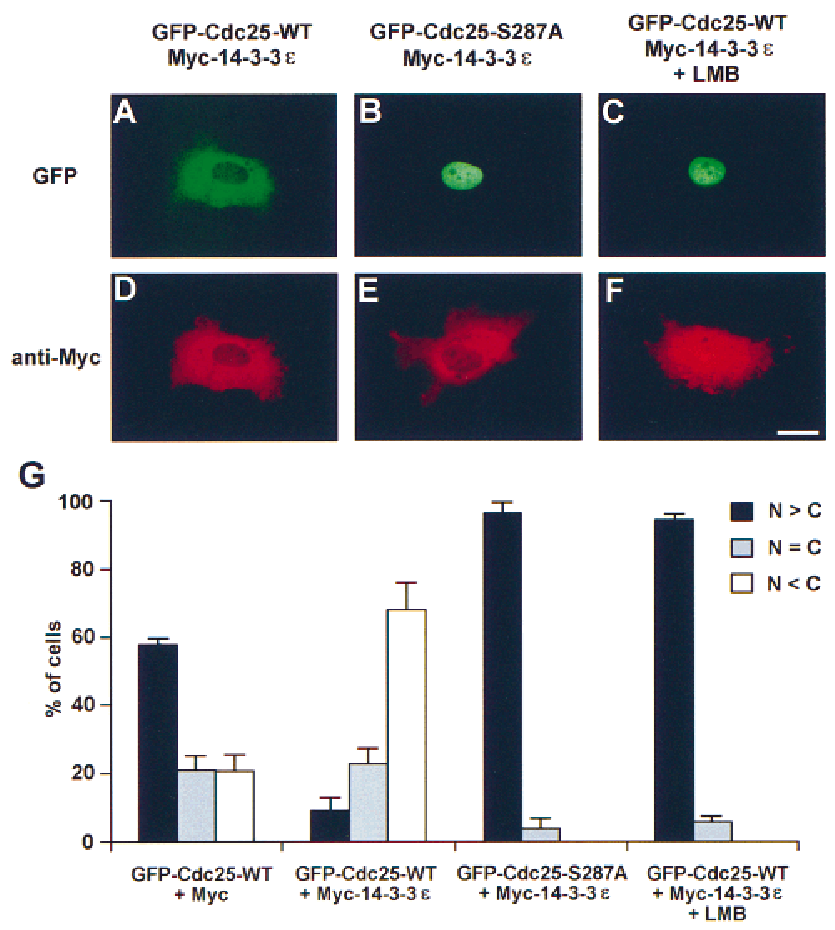

Figure 2. Coexpression of Myc-14-3-3€ and GFP-Cdc25 proteins in XTC cells. Cells were cotransfected with plasmids encoding Myc-tagged 14-3-3€ $(A-F)$ and either GFP-Cdc25-WT $(A, C, D, F)$ or GFP-Cdc25-S287A $(B, E)$. After $16 \mathrm{hr}$, one set of cells was treated with $200 \mathrm{nM}$ LMB for $2 \mathrm{hr}$. At $18 \mathrm{hr}$ posttransfection, cells were stained with anti-Myc antibodies (9E10) and Texas Red-conjugated goat anti-mouse antibodies. Cells were observed for fluorescence of either GFP $(A-C)$ or Texas Red $(D-F)$. Magnification bar, $20 \mu \mathrm{m}$. (G) Quantitation of the results in $A-F$. In addition, the localization of GFP-Cdc25-WT in cells cotransfected with a plasmid encoding the Myc epitope alone (pMyc) was quantitated. The localization of the various GFPCdc25 proteins was scored according to whether it was higher in the nucleus, evenly distributed between the nucleus and cytoplasm, or higher in the cytoplasm. Results are the mean \pm S.D. from three experiments. (N) Nuclear; (C) cytoplasmic. 
Leu ( $X$ is any amino acid), but the hydrophobic residues Ile, Met, Phe, and Val can also be accommodated, and variations of this spacing have been reported (Mattaj and Englmeier 1998). Within Xenopus Cdc25C, there are a number of amino acid segments that resemble this con-

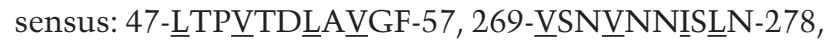

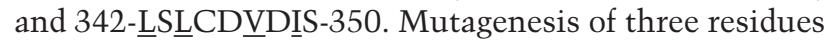
(Val-272, Ile-275, and Leu-277) in the 269-278 segment to alanine had no effect on the localization of the corresponding GFP-Cde25 mutant (not shown). In contrast, the 47-57 and 342-350 segments appear to have a role in the localization of Cdc25. As shown in Figure 3, alterations of Leu-53, Val-55, and Phe-57 to alanine resulted in a mutant (GFP-Cdc25-N1) that was mainly nuclear in the presence of Myc-14-3-3€. In particular, the GFPCdc25-N1 protein was more concentrated in the nucleus in $88 \%$ of transfected cells. Furthermore, no cells were detected in which GFP-Cdc25-N1 was predominantly cytoplasmic. Mutagenesis of Leu-344, Val-347, and Ile349 in the 342-350 segment to alanine also had a significant, but less severe, effect on the localization of the resulting mutant protein, which we called GFP-Cdc25N2. In particular, in the presence of Myc-14-3-3€, GFPCdc25-N2 was mainly cytoplasmic in only $13 \%$ of the cells as opposed to $68 \%$ of cells for the GFP-Cdc25-WT protein. Similarly, GFP-Cdc25-N2 was mainly nuclear in $44 \%$ of the cells, whereas only $9 \%$ of the cells contained GFP-Cdc25-WT mainly in the nucleus.

We then prepared a combination mutant (GFP-Cdc25N1N2) in which both the 47-57 and 342-350 segments were altered (Fig. 3). We observed that the sextuple GFPCdc25-N1N2 mutant had a more pronounced defect than either triple mutant alone. The GFP-Cdc25-N1N2 protein was mainly nuclear in $95 \%$ of cells coexpressing Myc-14-3-3€. Furthermore, in most of these cells, essentially no signal from GFP could be detected in the cytoplasm, which contrasts with the localization of the GFPCdc25-N1 and GFP-Cdc25-N2 proteins (Fig. 3B,C,F). We also compared the localization of Myc-14-3-3€ in cells coexpressing either GFP-Cdc25-WT or GFPCdc25-N1N2. As shown in Figure 3, D and E, some of the Myc-14-3-3€ accumulates in the nucleus along with GFP-Cdc25-N1N2, whereas there is essentially no nuclear Myc-14-3-3 $\epsilon$ in cells coexpressing GFP-Cdc25-WT.

We also examined the possibility that $14-3-3 \epsilon$ contains an NES that it could provide by binding to Cdc25. Xenopus $14-3-3 \epsilon$ contains at least two hydrophobic stretches that have characteristics of an NES (199-FDDAIAELDTLS-210 and 219-LIMQLLRDNLTLW-231), although the 199-210 segment is a relatively poor match. We prepared the following mutants of Myc-14-3-3€ in which two or more of these hydrophobic residues were mutated to alanine: M1 (Leu-206 and Leu-209); M2 (Leu228 and Leu-230); M3 (a combination of M1 and M2); M4 (a sextuple mutant of Leu-219, Ile-220, Leu-223, Leu-224, Leu-228, and Leu-230); and M5 (Ile-220 and Leu-224). The M5 mutant corresponds to the Rad24-nes mutant of the fission yeast 14-3-3 homolog Rad24, which was recently reported to be defective for nuclear export (LopezGirona et al. 1999). As shown in Table 1, the M1, M2, and M3 mutants were as effective as wild-type Myc-14$3-3 \epsilon$ in eliciting the cytoplasmic retention of GFPCdc25-WT. In contrast, the M4 and M5 mutants could not promote the cytoplasmic retention of GFP-Cdc25-WT.

In previous studies, several of the conserved hydrophobic residues within helix 9 of 14-3-3, which corresponds to the 219-231 segment of Xenopus 14-3-3€, have been implicated in the binding of 14-3-3 to its ligands (Liu et al. 1995; Yaffe et al. 1997; Thorson et al. 1998; Wang et al. 1998). To evaluate whether the M4 and M5 mutants could bind to Cdc25, we mixed wild-type His6-14-3-3€, His6-14-3-3€-M4, or His6-14-3-3€-M5 with the GSTCdc25-WT protein in the presence of diluted interphase extract from Xenopus eggs (a source of kinase activity for Ser-287 of Cdc25). After reisolation of GST-Cdc25-WT with glutathione beads and immunoblotting with anti14-3-3€ antibodies, we observed that binding of the M4 and M5 mutants of His6-14-3-3€ to Cdc25 was negligible in comparison with the binding of wild-type His6-14-3$3 \epsilon$ (Fig. 4A). To address the possibility that the nuclear accumulation of the N1N2 mutant of Cdc25 might be attributable to a defect in its ability to associate with 14-3-3€, we performed the following binding experiment.
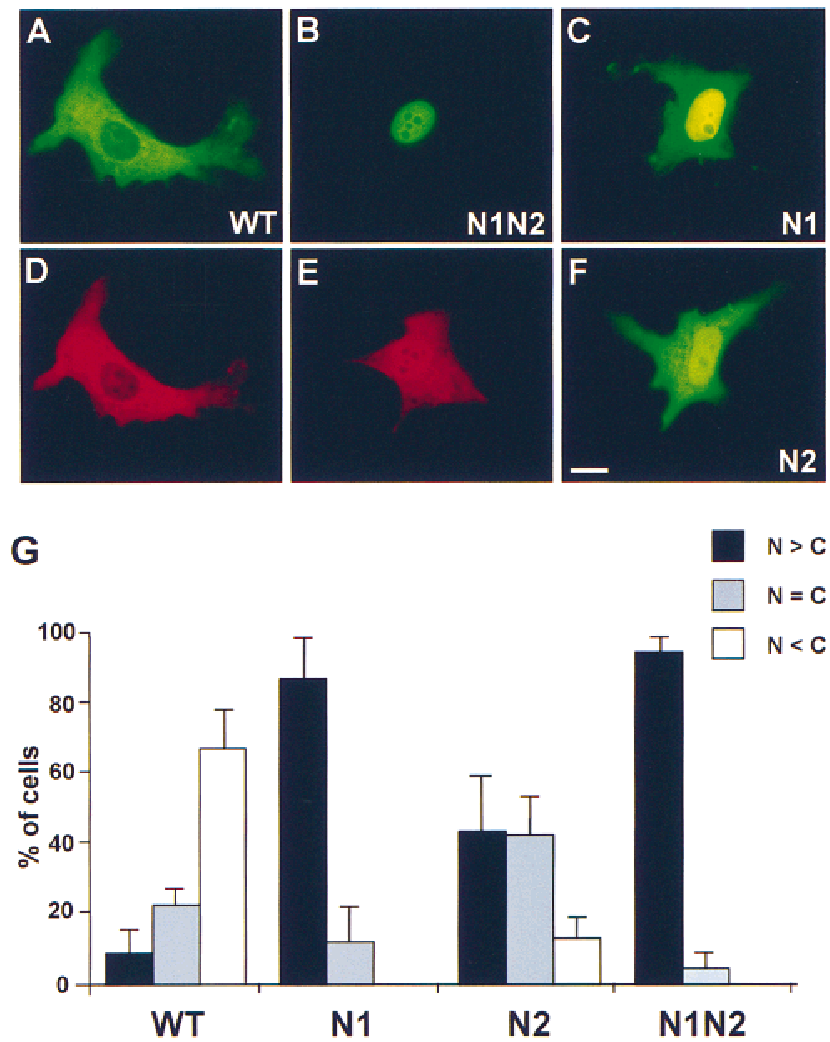

Figure 3. Mutagenesis of nuclear export sequences in Xenopus Cdc25. Plasmids encoding GFP-Cdc25-WT $(A, D)$, GFP-Cdc25N1N2 $(B, E)$, GFP-Cdc25-N1 $(C)$, or GFP-Cdc25-N2 $(F)$ were cotransfected into XTC cells with pMyc-14-3-3€. Cells were processed for visualization of GFP $(A, B, C, F)$ and the Myc epitope $(D, E) .(G)$ The localization of the various GFP-Cdc25 proteins was quantitated (mean \pm S.D. for two experiments) as described in the legend to Fig. 2. 
Table 1. Effect of Myc-14-3-3e proteins with mutations in conserved hydrophobic residues on the localization of the GFP-Cdc25-WT protein

\begin{tabular}{|c|c|c|c|}
\hline & $\begin{array}{c}\text { Nuclear }>\text { cytoplasmic } \\
(\%)\end{array}$ & $\begin{array}{c}\text { Nuclear }=\text { cytoplasmic } \\
(\%)\end{array}$ & $\begin{array}{c}\text { Nuclear }<\text { cytoplasmic } \\
(\%)\end{array}$ \\
\hline pEGFP-Cdc25-WT + pMyc-14-3-3e-M1 & $0.7 \pm 0.7$ & $21.4 \pm 7.8$ & $78.0 \pm 7.2$ \\
\hline pEGFP-Cdc25-WT + pMyc-14-3-3e-M2 & $1.7 \pm 0.2$ & $18.3 \pm 0.5$ & $80.0 \pm 0.8$ \\
\hline pEGFP-Cdc25-WT + pMyc-14-3-3e-M3 & $2.3 \pm 2.3$ & $22.9 \pm 0.2$ & $74.8 \pm 2.5$ \\
\hline pEGFP-Cdc25-WT + pMyc-14-3-3e-M4 & $63.2 \pm 2.9$ & $21.9 \pm 0.5$ & $14.9 \pm 2.4$ \\
\hline pEGFP-Cdc25-WT + pMyc-14-3-3e-M5 & $56.8 \pm 5.9$ & $26.3 \pm 0.8$ & $16.9 \pm 6.7$ \\
\hline
\end{tabular}

XTC cells were transfected with the indicated plasmids and processed after $18 \mathrm{hr}$ to localize the GFP-Cdc25-WT protein by fluorescence microscopy. The localization of the GFP-Cdc25-WT protein was scored according to whether it was higher in the nucleus, evenly distributed between the nucleus and cytoplasm, or higher in the cytoplasm. Results are the mean \pm S.D. from two independent experiments.

Bacterially expressed GST-Cde25-WT, GST-Cdc25N1N2, and GST-Cdc25-S287A proteins were mixed with His6-14-3-3€ in diluted interphase egg extract. After reisolation of the GST-Cdc25 proteins and immunoblotting with anti-14-3-3€ antibodies, we found that His6-14-3-3€ bound well to both GST-Cdc25-WT and GST-Cdc25-N1N2 (Fig. 4A). As expected, there was no binding to the S287A mutant.

Finally, to explore the possibility that 14-3-3 affects the recognition of Cdc25 for nuclear import, we asked whether the binding of importin- $\alpha$, a receptor for a bipartite NLS (Mattaj and Englmeier 1998; Moore et al. 1999|, to Cdc25 would be affected by 14-3-3. For this purpose, we incubated GST-importin- $\alpha$ in interphase Xenopus egg cytosol in the presence of ${ }^{35}$ S-labeled His6Cdc25-WT or His6-Cdc25-S287A. Under these conditions, endogenous 14-3-3 in the egg extracts binds efficiently to the radiolabeled His6-Cdc25-WT but not His6-Cdc25-S287A (not shown). After reisolation of GST-importin- $\alpha$ with glutathione-agarose, we observed that the binding of ${ }^{35}$ S-His6-Cdc25-WT to GST-importin- $\alpha$ was significantly reduced in comparison with ${ }^{35} \mathrm{~S}-$ His6-Cdc25-S287A (Fig. 4B,C).

\section{Discussion}

In this report we have examined the role of $14-3-3$ proteins in regulating the localization of the mitosis-inducing phosphatase Cdc25. One observation is that the S287A mutant of Xenopus Cdc25 that cannot bind 143-3 readily accumulates in the nuclear compartment of transiently transfected XTC cells. In contrast, wild-type Cdc25 with an intact 14-3-3 binding site is mainly cytoplasmic, provided that there is an adequate supply of 14-3-3 available in the same cell. The entry of the S287A mutant of Xenopus Cde25 into the nucleus involves recognition of its NLS by the importin-mediated nuclear import pathway (Mattaj and Englmeier 1998), because Cdc25 binds well to importin- $\alpha$ and disruption of its NLS completely abrogates nuclear localization.

Another finding is that inhibition of nuclear export perturbs cytoplasmic retention of wild-type Cdc25. In particular, LMB elicits a rapid redistribution (within 2 hr) of Cdc25 from the cytoplasm to the nucleus. Because LMB acts by inhibiting Crm1/Exportin 1, the receptor for an NES, it appears that one or more proteins must be continually exported from the nucleus for Cdc25 to remain properly localized to the cytoplasm during interphase. Mutagenesis studies have indicated that Xenopus Cdc25 contains two segments (residues 47-57 and 342350 ) that both resemble a classical NES structurally and play a role in the cytoplasmic localization of Cdc25. A triple mutant in the first segment (N1) has a stronger phenotype than the triple N2 mutant, and the combined N1N2 mutant is essentially absent from the cytoplasm in cells coexpressing Myc-14-3-3€.

Although Xenopus 14-3-3€ also contains one or more regions that resemble an NES, various mutants of Myc$14-3-3 \epsilon$ with alterations in conserved hydrophobic residues in these regions (helices 8 and 9) either can still

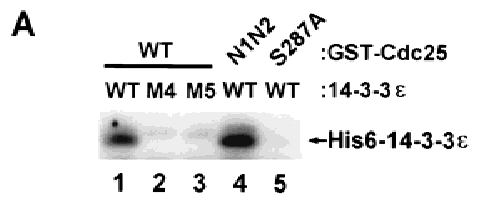

B
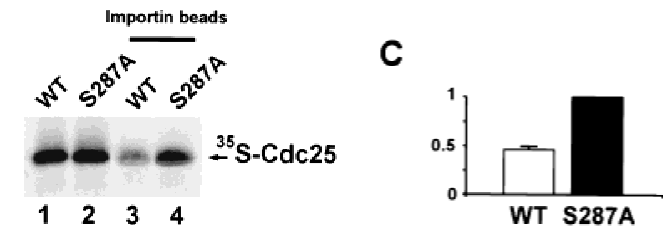

Figure 4. Characterization of the binding between Cdc25 and 14-3-3є. (A) Wild-type His6-14-3-3€ (lanes 1,4,5), His6-14-3-3€M4 (lane 2), His6-14-3-3є-M5 (lane 3), GST-Cdc25-WT (lanes 1-3), GST-Cdc25-N1N2 (lane 4), or GST-Cdc25-S287A (lane 5) were added to diluted interphase egg extract for $30 \mathrm{~min}$. The GST-Cdc25 proteins were isolated with glutathione beads and immunoblotted with anti-14-3-3€ antibodies to detect His6-14$3-3 \epsilon$. Under these conditions, the binding of endogenous 14-3-3€ (which has a faster electrophoretic mobility) is not detectable. (B) Binding of 14-3-3 hinders association of Cdc25 with importin- $\alpha .{ }^{35}$ S-Labeled His6-Cdc25-WT (lane 1) and His6-Cdc25S287A (lane 2) were incubated in interphase cytosol containing GST-importin- $\alpha$. GST-importin- $\alpha$ was isolated with glutathione-agarose, and binding of radiolabeled His6-Cdc25-WT (lane 3) and His6-Cdc25-S287A (lane 4) was assessed. (C) Quantitation in arbitrary units of data from lanes 3 and 4 of $B$ (mean \pm S.D. from three experiments). 
elicit cytoplasmic localization of Cde25 (M1, M2, M3) or are unable to bind to Cdc25 (M4, M5). The M5 protein contains mutations in helix 9 corresponding to those in the Rad24-nes mutant that was reported to be defective for nuclear export in fission yeast (Lopez-Girona et al. 1999|. We find that wild-type Myc-14-3-3€ cannot promote the nuclear exclusion of GFP-Cdc25-N1N2, despite the fact that Myc-14-3-3€ and GFP-Cdc25-N1N2 bind efficiently to one another. Furthermore, Myc-14-3$3 \epsilon$ and GFP-Cdc25-N1N2 accumulate together in the nucleus. These observations indicate that the NESs of Cdc25 are necessary for its exclusion from the nucleus and that the binding of $14-3-3 \epsilon$ is not sufficient for export of Cdc25. Our studies do not rule out the possibilities that helix 9 of $14-3-3 \epsilon$, in addition to being important for binding to Cdc25, could act cooperatively with the NESs of Xenopus Cde25 or serve as an NES to maintain a high cytoplasmic concentration of 14-3-3 $\epsilon$. In addition, binding of $14-3-3 \epsilon$ could alter the conformation of Cdc25 to favor recognition of the NESs in Cdc25.

Taken together, these results suggest a model whereby at least one function of 14-3-3 is to reversibly hinder the nuclear import of Cdc25. Consistent with this notion, binding of 14-3-3 inhibits the association of Cdc25 with importin- $\alpha$. Transient dissociation of $14-3-3 \epsilon$, perhaps followed by dephosphorylation of Ser-287, would result in nuclear translocation of Cdc25. Once in the nucleus, Cdc25 could have multiple fates. If the conditions were not appropriate for mitosis, Cdc25 could be exported directly back to the cytoplasm after recognition by nuclear export factors. In this case, if Cdc 25 did not associate with $14-3-3 \epsilon$ before export, it would be imported rapidly back into the nucleus. Conversely, if Cdc25 underwent phosphorylation in the nucleus by Ser-287-specific kinases such as Chk1 (Sanchez et al. 1997; Kumagai et al. 1998a), which might be favored during the operation of $\mathrm{G}_{2} / \mathrm{M}$ checkpoint, $14-3-3 \epsilon$ could rebind to $\mathrm{Cdc} 25$, and the resulting Cdc25/14-3-3€ complex would not be able to enter the nucleus again until dissociation of 14-3-3€. According to this scenario, Cdc25 would continually shuttle between the nucleus and cytoplasm, and binding of 14-3-3 would shift the equilibrium from a nuclear to cytoplasmic localization. If the conditions were appropriate for mitosis, Cdc 25 could undergo activation owing to phosphorylation of its amino-terminal regulatory domain. Conceivably, phosphorylation of Cde 25 by mitotic kinases such as $\mathrm{Cdc} 2 /$ cyclin $\mathrm{B}$ and $\mathrm{Plx} 1$ could affect recognition of Cdc25 for nuclear export, as is the case for cyclin B1 (Li et al. 1997; Yang et al. 1998). Interestingly, there are two mitotic phosphorylation sites (42-SPEQ-45 and 48-TPVT-51) in or near the N1 NES segment at residues 47-57 of Xenopus Cdc25. Inappropriate accumulation of Cdc25 in the nuclear compartment, as occurs when the 14-3-3 binding site in Cdc25 is mutated, may lead to an accelerated entry into mitosis and a derangement of checkpoint controls (Peng et al. 1997; Kumagai et al. 1998b). This model would not preclude the possibility that 14-3-3 proteins could have additional roles in regulating Cdc25, for example, by affecting its interactions with other Cdc25-regulatory factors.

\section{Materials and methods}

Preparation of plasmids for expression of proteins in XTC cells and Escherichia coli

The pEGFP-Cdc25-WT and pEGFP-Cdc25-S287A vectors for expression of the GFP-Cdc25-WT and GFP-Cdc25-S287A proteins in XTC cells were prepared as follows: The BglII-KpnI fragments from pBluescriptSK-Cdc25-1 and pFastBacHTa-Cdc25-S287A (Kumagai et al. 1998b) were produced by PCR using PfuTurbo (Stratagene) and the oligonucleotides Cdc25-BglII, CTGGAAGATCTATGGCAGAGAGTCACATAATG, and Cdc25-KpnI, CGGGGTACCTTAAAGCTTCATTATGCGGGC. The fragments were digested with $B g I I I$ and $K p n I$ and cloned into pEGFP-C1 (Clontech). pEGFP-Cdc25- NLS and pEGFP-Cdc25-S287A$\Delta$ NLS, each encoding a deletion of amino acids $311-316$, were prepared with the Quickchange kit using pEGFP-Cdc25-WT and pEGFP-Cdc25S287A, respectively, and the oligonucleotides CTAGACAGTGAAACGCCGGTGTCGACCAGCAGCTCCCTTCAACC and GGTTGAAGGGAGCTGCTGGTCGACACCGGCGTTTCACTGTCTAG. pEGFP_Cdc25N2 (encoding mutations L344A, V347A, and I349A) was produced with the Quickchange kit (Stratagene) using pEGFP-Cdc25-WT and the oligonucleotides CTCTTAAAAAGACGCTTTCTGCTTGTGATGCAGATGCCAGTACAGTTTTGGATG and CATCCAAAACTGTACTGGCATCTGCATCACAAGCAGAAAGCGTCTTTTTAAGAG. pEGFPCdc25-N1 (encoding mutations L53A, V55A, and F57A) and pEGFPCdc25-N1N2 (encoding mutations L53A, V55A, F57A, L344A, V347A, and I349A) were produced with the Quickchange kit using pEGFPCdc25-WT and pEGFP-Cdc25-N2, respectively, and the oligonucleotides CCTGTGACTGACGCTGCTGCAGGAGCTAGTAACCTAAGTACC and GGTACTTAGGTTACTAGCTCCTGCAGCAGCCAGTCAGTGTCC.

A vector for expression of Myc-tagged proteins in XTC cells was prepared as follows: pEGFP-C1 was digested with XhoI, followed by a partial digestion with $\mathrm{NcoI}$ to remove the GFP coding sequence, and ligated with the NcoI-XhoI fragment encoding the Myc epitope (MFQKLTSEEDL) from pCS-MT (Zhang et al. 1997) to generate pMyc. To generate pMyc$14-3-3 \epsilon$, the XhoI-KpnI fragment of Xenopus 14-3-3€ was produced by PCR with pET3-His6-14-3-3€ (Kumagai et al. 1998b) and the oligonucleotides 14-3E-XhoI, GAAGATCTCTCGAGATGGAAGAGCGAGAGGATTTAG, and 14-3E-KpnI, GGGGTACCTCACTGATTTTCATCTTCCAC. The XhoI-KpnI fragment of $14-3-3 \epsilon$ was cloned into pMyc digested with $X h o I$ and KpnI. pMyc-14-3-3€-M2 with mutations L228A and L230A was made by PCR using pET3-His6-14-3-3€ and the oligonucleotides 14-3E-Spe, CTATGGACTAGTGATATGCAAGGTGACGGTGAA; 14-3E-ATA, CATATCACTAGTCCATGCTGTCGCATTATCACGTAA; 14-3E-KpnI; and 14-3E-XhoI. pMyc-14-3-3€-M1 with mutations L206A and L209A and pMyc-14-3-3€-M3 with mutations L206A, L209A, L228A, and L230A were prepared with the Quickchange kit using pMyc-14-3-3€ and pMyc-14-3-3€-M2, respectively, and the oligonucleotides CGCCATTGCAGAAGCGGACACAGCGAGTGAAGAAAGTTAC and GTAACTTTCTTCACTCGCTGTGTCCGCTTCTGCAATGGCG. pMyc-14-3-3€-M4 with mutations L219A, I220A, L223A, L224A, L228A, and L230A was created by PCR using the oligonucleotides TATCGCGCGCCGCTTGCATGGCAGCTGTGGAGTCTTTG; ATTGGCGCGCGATAATGCGACAGCATGGACTTCAG; 14-3E-KpnI; and 14-3E-XhoI. pMyc-14-3-3€-M5 with mutations I220A and L224A was prepared with the Quickchange kit using the oligonucleotides CAAAGACTCCACACTCGCGATGCAATTGGCACGTGATAATCTGAC and GTCAGATTATCACGTGCCAATTGCATCGCGAGTGTGGAGTCTTG.

pGEX-Cdc25-N1N2 was produced by cloning the StuI-ClaI fragment of pEGFP-Cdc25-N1N2 into pGEX-Cdc25-WT digested with StuI and ClaI. pGEX-Cdc25-WT and pGEX-Cdc25-S287A and purification of GST-Cdc25 fusion proteins from Escherichia coli were described previously (Kumagai et al. 1998a). Xenopus GST-importin- $\alpha$ (Moore et al. 1999) was prepared by the same method. pET-His6-14-3-3€-M4 and pET-His6-14-3-3€-M5 were prepared by PCR using the M4 and M5 versions of pMyc-14-3-3€, respectively. His6-14-3-3€ proteins were prepared as described (Kumagai et al. 1998b).

Transfection of XTC cells

XTC cells were transfected using FuGENE 6 transfection reagent (Boehringer Mannheim Biochemicals) according to manufacturer's instructions and incubated for $18 \mathrm{hr}$. Typically, $\sim 5 \%$ of the cells expressed recombinant protein. Cells were fixed with $3 \%$ paraformaldehyde in phosphate-buffered saline and permeabilized with $0.5 \%$ Triton X-100. Myc epitope-tagged proteins were visualized with anti-Myc 9E10 monoclonal 
antibody (Calbiochem) and Texas Red-conjugated goat anti-mouse antibodies (Jackson Immunoresearch Laboratories).

Binding of Cdc25 to 14-3-3e

Glutathione-agarose beads containing GST-Cdc25-WT, GST-Cdc25N1N2, or GST-Cdc25-S287A were added to interphase extracts of Xenopus eggs that had been diluted 10 -fold with $80 \mathrm{~mm} \beta$-glycerolphosphate (pH 7.3), $20 \mathrm{~mm}$ EGTA, $15 \mathrm{~mm} \mathrm{MgCl}$, and $0.5 \%$ NP-40 containing an

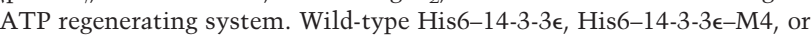
His6-14-3-3€-M5 were added to a concentration of $40 \mu \mathrm{g} / \mathrm{ml}$. After 30 min at $23^{\circ} \mathrm{C}$, beads were washed in dilution buffer and subjected to gel electrophoresis.

Binding of ${ }^{35}$ S-labeled Cdc25 proteins to GST-importin- $\alpha$

${ }^{35}$ S-Labeled His6-Cdc25-WT and ${ }^{35}$ S-labeled His6-Cdc25-S287A were radiolabeled for $7 \mathrm{hr}$ in $2 \times 10^{7}$ baculovirus-infected Sf9 cells in the presence of $0.5 \mathrm{mCi}$ of ${ }^{35} \mathrm{~S}$-Translabel (ICN) and purified as described (Kumagai and Dunphy 1996). ${ }^{35}$ S-Labeled His6-Cdc25-WT and His6-Cdc25S287A were incubated in the presence of GST-importin- $\alpha$ bound to glutathione-agarose in interphase Xenopus egg cytosol diluted 10-fold in a buffer containing $20 \mathrm{~mm}$ HEPES-KOH (pH 7.5), $80 \mathrm{~mm} \mathrm{NaCl}, 10 \mathrm{~mm}$ $\mathrm{MgCl}_{2}$, and an ATP regenerating system. After $30 \mathrm{~min}$ at $23^{\circ} \mathrm{C}$, the beads were washed in incubation buffer and subjected to gel electrophoresis.

\section{Acknowledgments}

We thank our colleagues for helpful comments. We are grateful to $\mathrm{M}$. Yoshida for leptomycin B and S. Kornbluth for the plasmid encoding GST-importin- $\alpha$. This work was supported in part by a grant from the National Institutes of Health (GM43974). W.G.D. is an investigator of the Howard Hughes Medical Institute.

The publication costs of this article were defrayed in part by payment of page charges. This article must therefore be hereby marked 'advertisement' in accordance with 18 USC section 1734 solely to indicate this fact.

\section{References}

Blasina, A., I. Van de Weyer, M. Laus, W. Luyten, A. Parker, and C. McGowan. 1999. A human homologue of the checkpoint kinase Cds1 directly inhibits Cdc25 phosphatase. Curr. Biol. 9: 1-10.

Dunphy, W.G. and A. Kumagai. 1991. The cdc25 protein contains an intrinsic phosphatase activity. Cell 67: 189-196.

Fornerod, M., M. Ohno, M. Yoshida, and I.W. Mattaj. 1997. CRM1 is an export receptor for leucine-rich nuclear export signals. Cell 90: 10511060.

Fukuda, M., S. Asano, T. Nakamura, M. Adachi, M. Yoshida, M. Yanagida, and E. Nishida. 1997. CRM1 is responsible for intracellular transport mediated by the nuclear export signal. Nature 390: 308 311.

Furnari, B., N. Rhind, and P. Russell. 1997. Cdc25 mitotic inducer targeted by Chk1 DNA damage checkpoint kinase. Science 277: 14951497.

Gautier, J., M.J. Solomon, R.N. Booher, J.F. Bazan, and M.W. Kirschner. 1991. cdc25 is a specific tyrosine phosphatase that directly activates p34cdc2. Cell 67: 197-211.

Hagting, A., C. Karlsson, P. Clute, M. Jackman, and J. Pines. 1998. MPF localization is controlled by nuclear export. EMBO J. 17: 4127-4138.

Hoffmann, I., P.R. Clarke, M.J. Marcote, E. Karsenti, and G. Draetta 1993. Phosphorylation and activation of human cde25-C by cde2cyclin B and its involvement in the self-amplification of MPF at mitosis. EMBO J. 12: 53-63.

Izumi, T. and J.L. Maller. 1995. Phosphorylation and activation of the Xenopus Cdc25 phosphatase in the absence of Cdc2 and Cdk2 kinase activity. Mol. Biol. Cell 6: 215-226.

Izumi, T., D.H. Walker, and J.L. Maller. 1992. Periodic changes in phosphorylation of the Xenopus cdc25 phosphatase regulate its activity. Mol. Biol. Cell 3: 927-939.

Jin, P., S. Hardy, and D.O. Morgan. 1998. Nuclear localization of cyclin B1 controls mitotic entry after DNA damage. J. Cell Biol. 141: 875885 .

Kumagai, A. and W.G. Dunphy. 1992. Regulation of the cdc25 protein during the cell cycle in Xenopus extracts. Cell 70: 139-151. - 1996. Purification and molecular cloning of Plx1, a Cdc25-regulatory kinase from Xenopus egg extracts. Science 273: 1377-1380.

Kumagai, A., Z. Guo, K.H. Emami, S.X. Wang and W.G. Dunphy. 1998a The Xenopus Chk1 protein kinase mediates a caffeine-sensitive pathway of checkpoint control in cell-free extracts. J. Cell Biol. 142: $1559-1569$.

Kumagai, A., P.S. Yakowec, and W.G. Dunphy. 1998b. 14-3-3 proteins act as negative regulators of the mitotic inducer Cdc25 in Xenopus egg extracts. Mol. Biol. Cell 9: 345-354.

Li, J., A.N. Meyer, and D.J. Donoghue. 1997. Nuclear localization of cyclin B1 mediates its biological activity and is regulated by phosphorylation. Proc. Nat1. Acad. Sci. 94: 502-507.

Liu, D., J. Bienkowska, C. Petosa, R.J. Collier, H. Fu, and R. Liddington. 1995. Crystal structure of the zeta isoform of the 14-3-3 protein. Nature 376: 191-194.

Lopez-Girona, A., B. Furnari, O. Mondesert, and P. Russell. 1999. Nuclear localization of Cdc25 is regulated by DNA damage and a 14-3-3 protein. Nature 397: 172-175.

Matsuoka, S., M. Huang, and S.J. Elledge. 1998. Linkage of ATM to cell cycle regulation by the Chk2 protein kinase. Science 282: 1893-1897.

Mattaj, I.W. and L. Englmeier. 1998. Nucleocytoplasmic transport: The soluble phase. Annu. Rev. Biochem. 67: 265-306.

Moore, J.D., J. Yang, R. Truant, and S. Kornbluth. 1999. Nuclear import of Cdk/Cyclin complexes: Identification of distinct mechanisms for import of Cdk2/Cyclin E and Cdc2/Cyclin B1. J. Cell Biol. 144: 213224.

Morgan, D.O. 1997. Cyclin-dependent kinases: Engines, clocks, and microprocessors. Annu. Rev. Cell Dev. Biol. 13: 261-291.

Ossareh-Nazari, B., F. Bachelerie, and C. Dargemont. 1997. Evidence for a role of CRM1 in signal-mediated nuclear protein export. Science 278: $141-144$

Peng, C.Y., P.R. Graves, R.S. Thoma, Z. Wu, A.S. Shaw, and H. PiwnicaWorms. 1997. Mitotic and G2 checkpoint control: Regulation of 14 3-3 protein binding by phosphorylation of Cdc25C on serine-216. Science 277: 1501-1505.

Sanchez, Y., C. Wong, R.S. Thoma, R. Richman, Z. Wu, H. PiwnicaWorms, and S.J. Elledge. 1997. Conservation of the Chk1 checkpoint pathway in mammals: Linkage of DNA damage to Cdk regulation through Cdc25. Science 277: 1497-1501.

Thorson, J.A., L.W. Yu, A.L. Hsu, N.Y. Shih, P.R. Graves, J.W. Tanner P.M. Allen, H. Piwnica-Worms, and A.S. Shaw. 1998. 14-3-3 proteins are required for maintenance of Raf-1 phosphorylation and kinase activity. Mol. Cell. Biol. 18: 5229-5238.

Toyoshima, F., T. Moriguchi, A. Wada, M. Fukuda, and E. Nishida. 1998 Nuclear export of cyclin B1 and its possible role in the DNA damageinduced G2 checkpoint. EMBO J. 17: 2728-2735.

Wang, H., L. Zhang, R. Liddington, and H. Fu. 1998. Mutations in the hydrophobic surface of an amphipathic groove of 14-3-3 zeta disrupt its interaction with Raf-1 kinase. J. Biol. Chem. 273: 16297-16304.

Yaffe, M.B., K. Rittinger, S. Volinia, P.R. Caron, A. Aitken, H. Leffers, S.J. Gamblin, S.J. Smerdon, and L.C. Cantley. 1997. The structural basis for 14-3-3: Phosphopeptide binding specificity. Cell 91: 961-971.

Yang, J., E.S. Bardes, J.D. Moore, J. Brennan, M.A. Powers, and S. Kornbluth. 1998. Control of cyclin B1 localization through regulated binding of the nuclear export factor CRM1. Genes \& Dev. 12: 2131-2143.

Zhang, Y., J.H. Chou, J. Bradley, C.I. Bargmann, and K. Zinn. 1997. The Caenorhabditis elegans seven-transmembrane protein ODR-10 functions as an odorant receptor in mammalian cells. Proc. Nat1. Acad. Sci. 94: 12162-12167. 


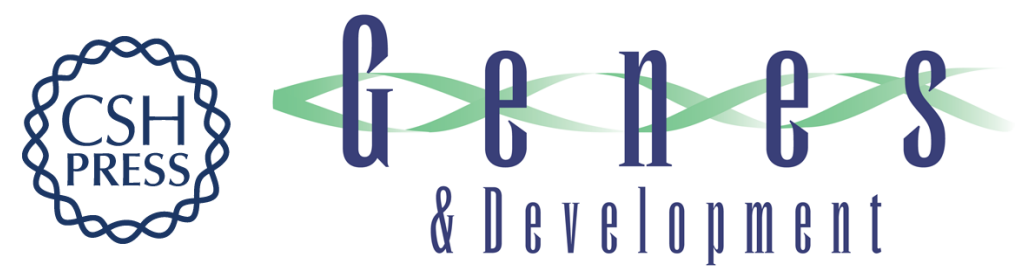

\section{Binding of 14-3-3 proteins and nuclear export control the intracellular localization of the mitotic inducer Cdc25}

Akiko Kumagai and William G. Dunphy

Genes Dev. 1999, 13:

References This article cites 31 articles, 19 of which can be accessed free at:

http://genesdev.cshlp.org/content/13/9/1067.full.html\#ref-list-1

License

Email Alerting Receive free email alerts when new articles cite this article - sign up in the box at the top Service right corner of the article or click here.

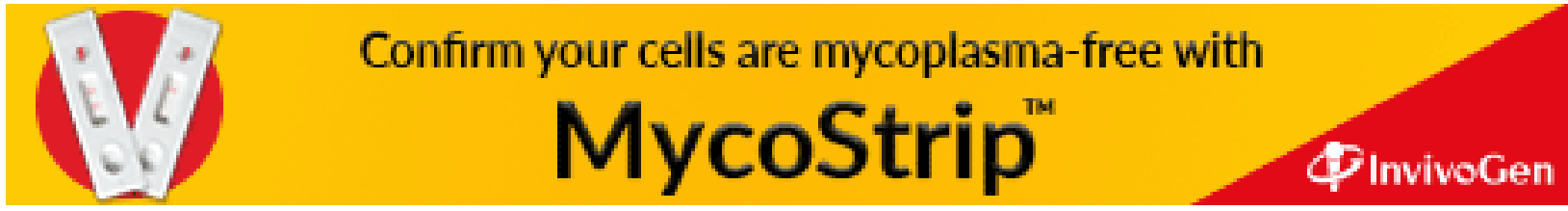

